\title{
FINAL REPORT:
}

DOE Award Number: DE-FG02-04ER63796

Title: Physical Properties of Hanford Transuranic Waste Sludge

Project period: 03/01/2004 - 11/30/2007

Principal Investor: John C. Berg

\section{PHYSICAL PROPERTIES OF HANFORD TRANSURANIC WASTE}

\author{
Prepared by: $\quad$ John C. Berg \\ Rehnberg Professor of Chemical Engineering \\ Box 351750 \\ University of Washington \\ Seattle, WA 98195
}

\begin{abstract}
The research described herein was undertaken to provide needed physical property descriptions of the Hanford transuranic tank sludges under conditions that might exist during retrieval, treatment, packaging and transportation for disposal. The work addressed the development of a fundamental understanding of the types of systems represented by these sludge suspensions through correlation of the macroscopic rheological properties with particle interactions occurring at the colloidal scale in the various liquid media. The results of the work have advanced existing understanding of the sedimentation and aggregation properties of complex colloidal suspensions. Bench scale models were investigated with respect to their structural, colloidal and rheological properties that should be useful for the development and optimization of techniques to process the wastes at various DOE sites.

The research resulted in the PhD Dissertation of one student, Dr. Peter B. Laxton, four publications in technical journals, and two presentations at national technical meetings. These items are summarized with full citations following a description of the scientific results obtained.
\end{abstract}




\section{OUTLINE OF REPORT}

I. Gel Trapping of Dense Colloids
A. Introduction
B. Methods
C. Results

II. Relating Clay Yield Stress to Colloidal Parameters
A. Introduction
B. Methods
C. Results

III. The Link between Micromechanical Bond Rigidity and Macroscopic Shear Moduli
A. Introduction
B. Methods
C. Results

IV. Colloid Aggregation Arrested by Caging within a Polymer Network
A. Introduction
B. Methods
C. Results

V. Summary of Deliverables 


\section{Gel Trapping of Dense Colloids}

\section{A. Introduction}

Phase density differences in dispersions often lead to sedimentation, causing problems for materials where spatial uniformity is important. The problem may be addressed through the use of rheology modifiers in the continuous phase. Weak polymer gels have found use for this purpose in many cases where they appear to be capable of trapping dispersoid particles in a three-dimensional matrix while displaying water-like viscosities at low shear. Attempts to predict sedimentation stability in terms of particle properties (size, shape, density difference) and gel yield stress have led to qualitative success for suspensions of large particles. Specifically, a dimensionless yield parameter $Y$ was defined as ${ }^{1}$ :

$$
Y=\frac{\tau_{\mathrm{y}}}{d(\Delta \rho) g},
$$

where $\tau_{\mathrm{y}}$ is the yield stress of the suspending medium, $d$ is the particle diameter, and $\Delta \rho$ is the density difference between the particles and the medium. Different investigators have found, experimentally, different critical values of $Y$ for sedimentation, with the most recent consensus putting it at $\approx 0.06 .{ }^{2}$ The effect of particle size, however, in particular the case in which colloidal dimensions are approached, had not been investigated, and the present work addressed itself to the determination of useful stability criteria for colloidal dispersions in terms of readily accessible viscoelastic descriptors.

\section{B. Methods}

Results were obtained for systems consisting of $12 \mu \mathrm{m}$ poly(methyl methacrylate) (PMMA) spheres dispersed in aqueous gellan gum. Aqueous gellan gum, Kelcogel (CPKelco, San Diego, CA), solutions, crosslinked with $\mathrm{KCl}$, were used as model suspending media. This material was ideal due to its controllable rheological properties.

\footnotetext{
${ }^{1}$ U.Ts. Andres, Sov. Phys. Dok1. 5 (1960) 723.

2 R.P. Chhabra, Bubbles, Drops, and Particles in Non-Newtonian Fluids, CRC Press, Boca Raton, 1993, p. 90.

A.N. Beris, J.A. Tsamopoulos, R.C. Armstrong, R.A. Bround, J. Fluid Mech. 158 (1985) 218.

L. Jossic, A. Magnin, AIChE J. 13 (1967) 1195.
} 
Low shear viscosity, $\eta_{0}$, high shear viscosity, $\eta_{\infty}$, and especially yield stress, $\tau_{\mathrm{y}}$, were all tunable through formulation. Polymer concentration was directlyrelated to $\eta_{\infty}$ : at a polymer concentration of $0.1 \mathrm{wt} \% \eta_{\infty}$ was about $0.003 \mathrm{~Pa} \mathrm{~s}$, and this viscosity increased with increasing concentration. Both $\tau$ y and $\eta 0$ depended on both polymer and salt concentration. At a given polymer concentration, $\tau_{\mathrm{y}}$ increased exponentially with $[\mathrm{KCl}]$. At some concentration $\tau_{\mathrm{y}}$ began to level off, corresponding to the concentration at which all crosslinkable sites are saturated. Gels were prepared by adding the gum as a powder to room temperature DI water in a beaker. Once the powder was sufficiently wet, shear was

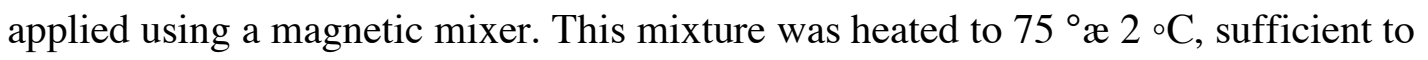
dissolve the polymer. Concentrated $\mathrm{KCl}$ solution was then added dropwise to the warm polymer solution until the desired concentration was achieved. Next, this mixture was allowed to cool to room temperature under shear. Once at room temperature the gel was split into $\sim 10 \mathrm{ml}$ aliquots in test tubes for later rheological and sedimentation studies. These samples were allowed to rest unperturbed for at least $18 \mathrm{~h}$ before they were used for any experiments. The solids used for sedimentation studies were $12 \mu \mathrm{m}$ diameter poly(methyl methacrylate) (PMMA) spheres which had a specific gravity of 1.19.

Rheological characterizations were performed at room temperature using a double-gap Couette flow geometry ona Bohlin VOR rheometer (Bohlin Reologi, Lund, Sweden). Shear sweeps were carried out in a controlled strain mode, typically in a range of $1.8 \times 10^{-3}$ to $1.5 \times 10^{2} \mathrm{~s}^{-1}$, primarily to determine yield stress. Oscillatory rheometry was also performed on the suspending media in order to investigate more fully their viscoelastic properties. The materials were placed in the gap between a $30 \mathrm{~mm}$ diameter, $5.4^{\circ}$ cone and plate. The plate was oscillated over angular amplitudes sufficiently small to avoid breakdown of the gel structure, i.e., keeping the material within its linear viscoelastic region, at frequencies ranging from 0.05 to $30 \mathrm{~Hz}$. The viscoelastic properties were quantified in terms of $G^{\prime}$ and $G^{\prime \prime}$, the real and imaginary components of the complex shear modulus of the material. $G^{\prime}$, the storage modulus gives the elastic nature of the gel, while $G^{\prime \prime}$, the loss modulus, gives the viscous nature. Both were obtained by instrument software that tracked the magnitude and phase lag of the torque on the cone for a given oscillatory frequency of the plate. Prior to performance of the frequency shear sweeps for each material, an amplitude strain sweep was performed at a frequency of $1 \mathrm{~Hz}$ to 
determine the limits of the linear viscoelastic region, as heralded by a sharp drop in $G^{\prime}$ at the point of breakdown. Allowable amplitudes were found up to $60-75 \%$ of the instrument maximum (corresponding to a peripheral displacement equal to the maximum gap width) for the different materials investigated. A common issue in the rheological characterization of gels is the shear history dependence of their properties. The problem was addressed in this work by following a fixed protocol. All samples for rheological or sedimentation testing were shaken by hand in their test tubes, and then each sample was aged for $10 \mathrm{~min}$ to allow it to recover some degree of structure before it was tested in an experiment. Repeating this procedure for multiple samples showed consistent shear sweep data. This repeatability gave assurance that the yield stress measured in the rheometer was the same as the yield stress of the samples used in subsequent centrifugation and sedimentation experiments in which the relevant gel age was 10 min. A second problem with the rheometry of gels is slip, caused by a depletion of the disperse phase near the wall of the measuring system. This is commonly overcome with either a roughened rheometer tool or a vane tool. Shear sweeps of a sample gel were run with a roughened cone and plate and a polished cone and plate in order to determine whether slip would be a significant problem. It was found that a smooth tool surface was satisfactory for measuring yield stress, the property sought in running the shear sweeps. Slip did produce a falsely low value for $\eta_{0}$ but did not affect the determination of $\tau_{\mathrm{y}}$. Sedimentation behavior of the latex spheres in the gels was observed with basic jar testing. PMMA spheres were suspended in a gellan sample and allowed to rest. Stable samples and samples that allowed sedimentation showed a clear difference. Suspensions left for $12 \mathrm{~h}$ either showed no visible change or allowed all solids to settle out. Every stable sample allowed to rest for extended periods continued to show stability several months after the initial dispersion was prepared. Samples that prevented gravitational sedimentation were spun in an International microcentrifuge (International Equipment Co., Needham Hts., MA) to induce settling. The centrifuge experiments were done on different samples of the same gel until the lowest speed that caused sedimentation was found. This speed was then used to calculate the critical yield parameter, with centrifugal acceleration on the particle replacing the gravitational acceleration in the equation for the critical yield parameter, $Y_{\mathrm{c}}$ : 


$$
Y_{\mathrm{c}}=\frac{\tau_{\mathrm{c}}}{\mathrm{d}(\Delta \rho) \omega_{\mathrm{c}}^{2} r}
$$

where $\omega_{\mathrm{c}}$ is the critical centrifuge rate and $r$ is the distance of a particle from the axis of rotation.

\section{Results}

Shear sweeps of gellan gels, shown in Fig. 5, suggested that these materials can be represented by the Herschel-Bulkley model with the Papanastasiou extension:

$$
\tau=\tau_{0}\left(1-e^{-m \dot{\gamma}}\right)+K(\dot{\gamma})^{n}
$$

where $\tau$ is the stress, $\dot{\gamma}$ is the strain rate, $\tau_{0}$ is the Bingham yield stress, $m$ is the stress growth exponent, Kis the consistency and $n$ is the power-law exponent. The HerschelBulkley model is a combination of the Bingham plastic and power-law models; above the yield stress a power-law equation is followed. Papanastasiou's extension allows for a finite viscosity below the yield stress, which is accounted for with the stress growth exponent. Small values of this exponent allow for small $\eta_{0}$ values, while values of $m>1000$ give essentially infinite $\eta 0$, as in an ideal Bingham plastic. This model fits the data well, the model fit to the data gives an $R^{2}=0.9986$. Typically, fitting parameters of $25<m<30,0.01<K<0.03,0.55<n<0.70$, and $\tau_{0} \approx \tau_{\text {y }}$ provided a good starting point for modeling the gels used in these experiments. A log-log plot of viscosity vs shear stress facilitated the location of the yield stress, which can be difficult from a stress-strain curve. From this type of plot the yield stress is the break point in the curve, where the viscosity begins to change sharply, usually over less than one order of magnitude in shear stress. Sedimentation experiments were performed using a number of gels with varying yield stress. Representative results from this set of experiments are summarized in Table 1 which reveals systems allowing sedimentation to occur with $Y$ values more than an order of magnitude greater than the predicted and previously measured critical value. The major distinction between this set of experiments and those done in the past is the size of the suspended solids. Although these spheres were $\sim 10^{3}$ times smaller than those used in earlier studies, they were not small enough to slip through gaps in the gel network. Using a conservative estimate for correlation length, $\xi$, which gives an idea of the size of gaps in a gel network, it was found that the gels in this study had a mesh size no larger than 0.1 
Table 1: Sedimentation experiment results. High $Y$ value systems allowed sedimentation when $\tan \delta \geq 1$

\begin{tabular}{llll}
\hline$\tau_{\mathrm{y}}(\mathrm{Pa})$ & $\tan \delta$ & $Y$ & \\
\hline 0.004 & 2.00 & 0.21 & sedimented \\
0.009 & 1.25 & 0.43 & sedimented \\
0.024 & 1.04 & 1.29 & sedimented \\
0.026 & 1.00 & 1.40 & sedimented \\
0.03 & 1.00 & 1.61 & sedimented \\
0.034 & 0.71 & 1.83 & stable \\
0.063 & 0.91 & 3.38 & stable \\
0.092 & 0.85 & 4.94 & stable \\
0.109 & 0.74 & 5.85 & stable \\
0.111 & 0.58 & 5.96 & stable \\
0.212 & 0.40 & 11.39 & stable \\
0.257 & 0.51 & 13.80 & stable \\
0.49 & 0.90 & 26.32 & stable \\
\hline
\end{tabular}

$\mu \mathrm{m}$, while the solids studied here were two orders of magnitude larger than that. This ruled out the possibility of solids falling through the network. A result of using small solids is the low yield stress required to suspend these solids against gravity. The suspending media in this work had two to three orders of magnitude lower yield stresses than the gels used in previous investigations. These low yield stress materials were approaching the lower limits of gelation which can be determined from oscillatory rheometry. Frequency sweeps gave storage and loss modulus as a function of oscillatory frequency. These plots illustrate that some samples were dominated by their elastic behavior while others were more viscous in nature. This difference in behavior can be quantified by the loss tangent, $\tan \delta$, which is the ratio of the loss to the storage modulus:

$$
\tan \delta=\frac{G^{\prime \prime}}{G^{\prime}} .
$$

The higher $\tan \delta$, the more liquid-like the sample, with a value of one considered to be a threshold between liquid and gel behavior. A second look at Table 1 shows that all samples that exhibited sedimentation had a $\tan \delta \geq 1$, calculated for the oscillatory frequency of $1 \mathrm{~Hz}$. This frequency was chosen somewhat arbitrarily; a low value was 
used in order to approach the case of trapped particles. The value of $1 \mathrm{~Hz}$ was sufficiently low while still allowing for satisfactorily reproducible results. Suspending media that seem to have sufficient yield stress will allow sedimentation unless their elastic nature dominates their viscous nature. This result was overlooked in studies where large spheres were suspended, since the materials near the critical yield parameter were much stronger.

All of the available samples that prevented sedimentation had a $\tan \delta<1$ as well as $Y$ values greater than previously computed $Y_{\mathrm{c}}$ values. A centrifuge was used in order to induce sedimentation in these samples. It was found that the $Y_{\mathrm{c}}$ for these samples, as calculated by locating $\omega_{\mathrm{c}}$, was in good agreement with the earlier experimental work of Atapattu et al. ${ }^{3} Y_{\mathrm{c}}=0.06 \pm 0.02$ was the average of eight experiments with eight gels of different yield stress, with the error indicated being one standard deviation, as shown in Table 2. Once the elasticity requirement is met, $Y_{\mathrm{c}}$ for this colloidal system is the same as the $Y_{\mathrm{c}}$ found for large solid particles.

Table 2: Centrifuge experiment results: $Y_{\mathrm{c}}$ agrees well with previous results when the $\tan \delta$ requirement is satisfied.

\begin{tabular}{ll}
\hline$\tau_{\mathrm{y}}(\mathrm{Pa})$ & $Y_{\mathrm{c}}$ \\
\hline 0.05 & 0.06 \\
0.08 & 0.06 \\
0.12 & 0.05 \\
0.15 & 0.04 \\
0.17 & 0.08 \\
0.18 & 0.08 \\
0.50 & 0.08 \\
0.64 & 0.09 \\
Average & 0.06 \\
Standard deviation & 0.02 \\
\hline
\end{tabular}

In summary, a combination of sedimentation and rheometric studies were performed on systems of $12 \mu \mathrm{m}$ PMMA spheres suspended in aqueous gellan gum gels. These experiments were performed in order to ascertain whether the critical yield

\footnotetext{
${ }^{3}$ D.D. Atapattu, R.P. Chhabra, P.H.T. Uhlherr, J. Non-Newtonian Fluid Mech. 59 (1995) 245.
} 
parameter as determined for macroscopic solids could be used to predict sedimentation stability for colloidal solids. It was found that the critical yield parameter for the system tested was within the experimental error of the $Y c$ as predicted for macroscopic solids. However, it was found that although the condition of $Y \geq Y_{\mathrm{c}}$ is necessary, it is not sufficient to predict sedimentation stability. A second requirement for predicting the sedimentation stability of a system is that the suspending medium must have $\tan \delta<1$. This requirement was always satisfied in previous experimental investigations with large spheres and therefore overlooked. These two conditions may yet be insufficient to always predict stability. For even smaller-sized suspended solids, a maximum gel network correlation length in relation to particle size may prove to be an additional requirement.

\section{Relating Clay Yield Stress to Colloidal Parameters}

\section{A. Introduction}

Aqueous systems of clays behave rheologically similarly to radioactive waste materials generated during the purification of weapons grade plutonium. ${ }^{4} \mathrm{~A}$ full understanding of how this material will behave during processing is necessary in order to transport and more securely contain it. Since radioactive materials are dangerous, difficult, and expensive to work with, a study of clay behavior was performed in order to approximate actual waste behavior. The goal was to predict rheological behavior based on accessible and controllable system parameters.

The mechanical properties of gels can be related to particle-particle interaction potentials, and in particular, a relationship of the yield stress to the interaction potentials has been proposed for spherical particles. ${ }^{5}$ These potentials, $\Phi$, can be differentiated to yield interparticle forces, $F$, which have a value of zero at potential minima. In a state of static equilibrium, colloidal systems will exist at interparticle separation distances, $D$, where the interaction potential is at a minimum, or where the force due to particle

\footnotetext{
${ }^{4}$ S.D. Rassat, L.M. Bagaasen, L.A. Mahoney, R.L. Russell, D.D. Caldwell, D.P. Mendoza, Physical and Liquid Chemical Simulant Formulations for Transuranic Wastes in Hanford Single-Shell Tanks, PNNL14333, National Technical Information Service, U.S. Dept. of Commerce, Springfield, VA p. iv.

${ }^{5}$ R.G. Larson, The Structure and Rheology of Complex Fluids, Oxford Univ. Press, New York, 1999, p. 351.
} 
interactions, $F\left(D_{0}\right)$, is zero. If a bulk sample in static equilibrium is then strained it has the effect of changing the particle separation distance, thus making the interparticle forces non-zero. When the strain is removed the system will relax to the potential minimum, where force is again zero. Following the potential vs distance curve to greater separation distances the slope reaches a maximum. Beyond this separation distance, $D_{y}$, the interparticle forces decrease as separation distance increases and the bulk system is said to yield. The bulk property yield stress, $\tau_{y}$, is thus proportional to the interparticle force at this separation distance times the number of particle contacts per unit area. The number of particle contacts scales as $\phi^{2} / a^{2}$, where $\phi$ is the volume fraction of the solids and $a$ is the particle radius, so that

$$
\tau_{\mathrm{y}} \propto \frac{\phi^{2}}{a^{2}} F\left(D_{y}\right)
$$

and since $F\left(D_{y}\right)=\Phi^{\prime}\left(D_{y}\right)$ it can be estimated as $-\Phi\left(D_{0}\right) / D_{0}$, so that

$$
\tau_{\mathrm{y}} \propto-\frac{\phi^{2}}{a^{2}} \frac{\Phi\left(D_{0}\right)}{D_{0}} .
$$

From DLVO theory,

$$
\Phi(D)=-\frac{a A_{H}}{12 D}+2 \pi \varepsilon \varepsilon_{0} a \zeta^{2} \ln \left[\frac{1}{1-\exp (-\kappa D)}\right],
$$

where $A_{H}$ is the Hamaker constant, $\varepsilon$ is the dielectric constant and $\varepsilon_{0}$ the permittivity of free space, $\zeta$ is the zeta potential and $\kappa$ is the Debye parameter. Combining Eqs. (6) and (7) gives:

$$
\tau_{y} \propto \frac{\phi^{2}}{a}\left(\frac{A_{H}}{12 D_{0}^{2}}-\frac{2 \pi \varepsilon \varepsilon_{0}}{D_{0}} \xi^{2} \ln \left[\frac{1}{1-\exp (-\kappa D)}\right]\right) .
$$

This inverse dependence of the yield stress of percolated colloids of alumina, titania and zirconia on the square of their respective zeta potentials has been reported.

It is generally accepted that most clay particles are platelets with a negative face charge and a positive edge charge under most $\mathrm{pH}$ conditions. Clays are therefore thought to set up a gel where the positively charged particle edges are attracted to the negative faces in a "house of cards" conformation. Clays forming electrostatically attractive gels 
under certain conditions of $\mathrm{pH}$, salt concentration, and solids loading would not behave as described by the proposed relation of yield stress to salt content for systems that develop microstructure through repulsive interactions, i.e., Eq. (8). It was therefore the goal of this work to develop a relationship of attractive clay gel yield stress to accessible colloidal properties and test this relationship against real systems.

Clays forming electrostatically attractive gels under certain conditions of $\mathrm{pH}$, salt concentration, and solids loading would not behave as described by the proposed relation of yield stress to salt content for systems that develop microstructure through repulsive interactions, i.e., Eq. (8). It was therefore the goal of this phase of the work to develop a relationship of attractive clay gel yield stress to accessible colloidal properties and test this relationship against real systems.

\section{B. Methods}

The net zeta potential for clays is presumably a composite of the edge and face zeta potentials. For a system of given surface charge, i.e., constant $\mathrm{pH}$ for the edge charge, the net zeta potential scales with ion content. Using indifferent electrolyte to adjust zeta potential should cause the edge and face chargeto scale proportionally with the net zeta potential. This would allow one to write the electrostatic component of the particle-particle interaction (i.e., he second term on the rhs of Eq. (8)) as

$$
\Phi_{e} \propto 2 \pi \varepsilon \varepsilon_{0} a \zeta(-\zeta) \ln \left[\frac{1}{1-\exp (-\kappa D)}\right] .
$$

Equation (8) would then remain unchanged, except that the sign of the second term on the right would be positive rather than negative. Systems displaying this behavior should have a fairly constant yield stress at low zeta potential where van der Waals forces dominate. Then as zeta potential becomes significant yield stress would increase with squared zeta potential, which would be very different from observations obtained for simpler oxides.

The model system in this study was the synthetic clay Laponite RD (Laporte Industries, Cheshire, UK) dispersed inaqueous $\mathrm{NaNO}_{3}$ solutions. Laponite is a magnesium silicate with sodium binding individual platelets into stacks when dry. When dispersed this clay is in the form of $\sim 30 \mathrm{~nm}$ diameter by $\sim 1 \mathrm{~nm}$ thick platelets. 
Dispersions of this clay were prepared by adding clay to deionized water while stirring. These mixtures were allowed to sit for a minimum of one day to ensure stack exfoliation before concentrated salt solution was added. After soaking, a small sample of the dispersion was sonicated, and the average hydrodynamic radius of the sample particles was measured by photon correlation spectroscopy (PCS) to be $17 \pm 2 \mathrm{~nm}$. Stock $1.5 \mathrm{M}$ $\mathrm{NaNO}_{3}$ was prepared with deionized water and solid NaNO3, minimum 99.0\% (SigmaAldrich, St. Louis, MO). Salt solution was added to clay suspensions under agitation, generating samples with a $\mathrm{NaNO}_{3}$ concentration, $C_{s}$, range of $0-1000 \mathrm{mM}$. These systems equilibrated to a natural $\mathrm{pH}$ of 8.5 as measured by a $\Phi 31 \mathrm{pH}$ meter (Beckman Instruments, Inc., Fullerton, CA). Dispersions of a mixture of naturally occurring clays, high purity kaolinites and bentonites (Georgia Kaolin Company, Elizabeth, NJ), were prepared in the same manner. The average hydrodynamic radius of these solids as measured by PCS after soaking in DI water and sonication was $351 \pm 58 \mathrm{~nm}$. These systems equilibrated to a natural $\mathrm{pH}$ of 6.7. All samples were allowed to age for at least three days before any measurements were made. This wait period was used in order to ensure reproducibletrends in the measured data.

Rheological characterizations were performed at a Peltier system controlled temperature of $25.0 \pm 0.1{ }^{\circ} \mathrm{C}$ with a Modular Compact Rheometer (MCR) 300 (Anton Paar, Ashland, VA). A 21-mm diameter, 16-mm height, 6-blade vane tool was submerged and rotated at a controlled rate in samples contained by a $23-\mathrm{mm}$ diameter cup. The vane tool was used in order to avoid slip effects that otherwise complicate rheological measurements of dispersions. Shear sweeps were carried out in controlled strain mode, in a range of $10-3-5 \pm 102 \mathrm{~s}^{-1}$, primarily to determine yield stress. Twentyfive separate shear rates were used to generate a rheogram with each shear rate held for $15 \mathrm{~s}$. In order to ensure that each sample had identical shear histories, each run began with a 60 -s preshear at a shear rate of $50 \mathrm{~s}^{-1}$ followed by a 15 -min rest period. The extended aging period followed by a preshear and subsequent wait period is common practice.

Zeta potential, $\zeta$, was determined using a Zeta-PALS (Brookhaven Instruments Corporation, Holtsville, NY). This instrument uses a derivative of laser Doppler 
electrophoresis, LDE, known as phase analysis light scattering, PALS. This technique provides for measurement of $\sim 10^{3}$ times smaller electrophoretic mobilities than LDE without complicating factors such as large voltages which result in sample heating and thermal convection currents. The greater sensitivity allows access to low electrophoretic mobility samples such as organosols or, in this work, high salt media. The electric field used to induce electrophoresis in these experiments had a strength of $1.35 \mathrm{~V} / \mathrm{cm}$ at a frequency of $2.0 \mathrm{~Hz}$. A $658-\mathrm{nm} 30-\mathrm{mW}$ laser was used as the light source with scattered light intensity measured at a scattering angle of $90^{\circ}$ by an avalanche photo-detector. Once electrophoretic mobility is measured, zeta potential is calculated using either the Smoluchowski or if necessary O'Brien and White analysis ${ }^{6}$ depending on system properties . Zeta-PALS software automatically returns a Smoluchowski-based zeta potential. When O'Brien and White analyses were necessary the software package MacMobility ${ }^{\circledR}$ (University of Melbourne, Australia) was used to compute zeta potential.

C. Results

Rheograms of $0.3 \mathrm{wt} \%$ laponite in various concentrations of $\mathrm{NaNO}_{3}$ were consistent with those often observed for gels. In general, weak gels exhibit a measurable and approximately constant low shear viscosity, $\eta_{0}$, followed by a sharp drop in apparent viscosity once the yield stress, $\tau_{y}$, is reached. Materials exhibiting this behavior are highly shear thinning. In this work $\tau_{y}$ was defined as the break point in the $\log -\log \eta-\tau$ curve, where viscosity begins to decrease sharply. Once beyond the yield point, the system reverts to rheological behavior typical of dispersions, in this case slightly shear thickening. $\tau_{y}$ was found to be a strong function of electrolyte concentration. In the case of $0.3 \mathrm{wt} \%$ laponite, $\tau_{y}$ was at a maximum at $C_{s} \leq 10 \mathrm{mM}$. As $C_{s}$ increases $\tau_{y}$ decreases until a minimum is reached near $C_{s}=100 \mathrm{mM}$. In high salt conditions, $C_{s}>100$, the change in $\tau_{y}$ is within the error of the technique. Another system property that is a strong function of $C_{s}$ is the zeta potential, with $|\zeta|$ decreasing as $C_{s}$ increases. Plotting $\tau_{y}$ vs $\xi^{2}$, as in Fig. 1, shows an increase in $\tau_{y}$ with $\zeta^{2}$. This was true in systems of both laponite and the mixture of naturalclays, kaolinites and bentonites, shown in Fig. 2.

The figures also show calculated yield stress as a function of zeta potential. In general

\footnotetext{
${ }^{6}$ R.W. O’Brien, L.R. White, J. Chem. Soc. Faraday Trans. 74 (1978) 1607.
} 


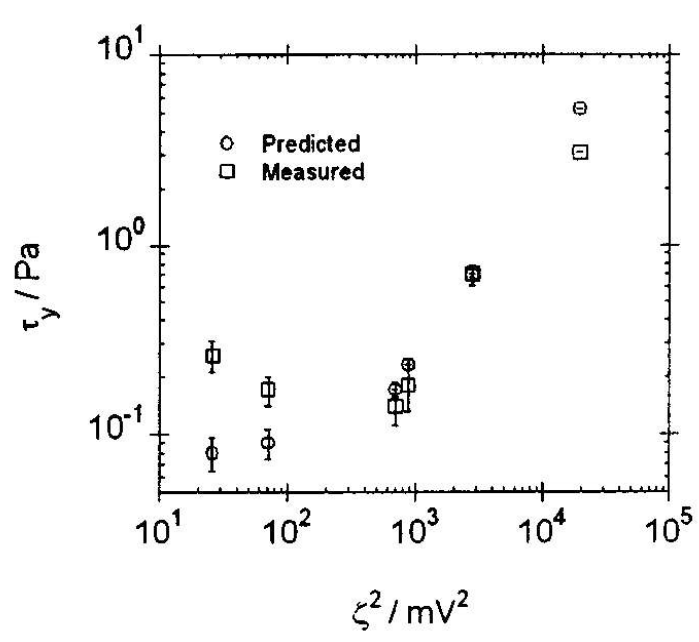

Fig. 1: Experimental and predicted $\tau_{y}$ vs $\zeta^{2}$ for $0.3 \mathrm{wt} \%$ laponite in solutions of various concentrations of $\mathrm{NaNO}_{3}$.

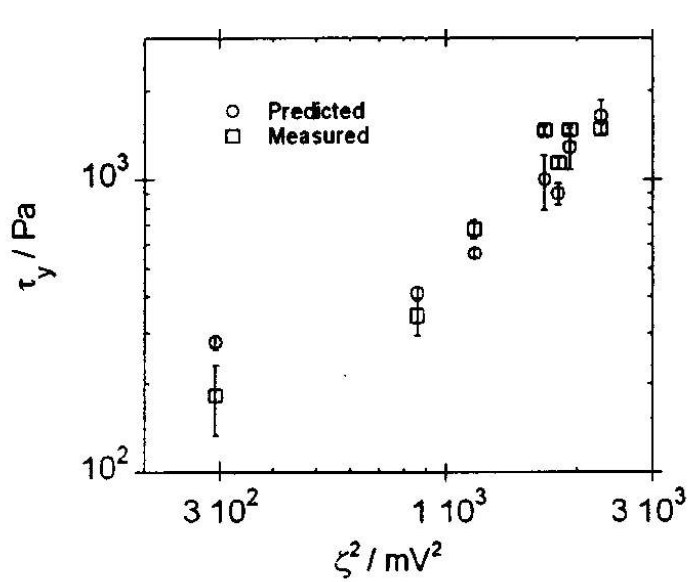

Fig. 1: Experimental and predicted $\tau_{y}$ vs $\xi^{2}$ for $15 \mathrm{wt} \%$ kaolinites and bentonites in solutions of various concentrations of $\mathrm{NaNO}_{3}$.

a reasonably constant yield stress at squared zeta potential values below about one hundred, beyond which yield stress increases linearly with square zeta potential. Figure 3 shows rheological behavior of a series of $1 \mathrm{wt} \%$ laponite in $10 \mathrm{mMNaNO} 3$ with various amounts of TSPP added to achieve a concentration range of 0-2.28 mM. TSPP is shown to reduce $\tau_{y}$ where a very small concentration of TSPP, $\sim 1 \mathrm{mM}$, completely removes the system yield stress. Above this TSPP concentration, the system reverts to behavior typically seen for dispersions of non-interacting particles, i.e., slightly shear thickening.

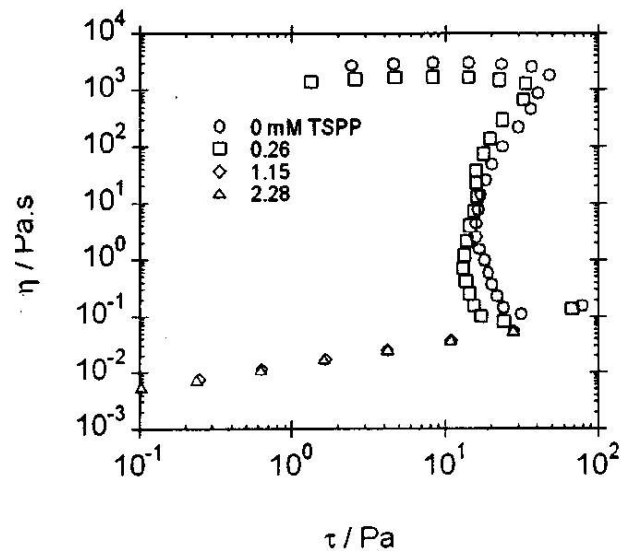

Fig. 3: $\tau_{y}$ vs $C_{\text {TSPP }}$ for $1 \mathrm{wt} \%$ laponite in 10 $\mathrm{mM} \mathrm{NaNO} \mathrm{N}_{3}$ with varying amounts of TSPP. 
Systems of aqueous clay dispersions have been shown to behave drastically differently than systems of homogeneously charged spheroidal colloids like titania and alumina. It is clear that clay systems do not follow the general relation found for such systems. This difference is due to two particle properties that make clays unique: disklike shape and heterogeneous charge distribution. Two models based on these particle properties have been used to describe the behavior of clay dispersions. One claims that the overwhelming negative face charge along with particle shape encourage a system where interparticle repulsions dominate, while the second argues that oppositely charged edge and face attract each other electrostatically setting up a gel. Others have proposed that both are true depending on system properties. In this work, where the $\mathrm{pH}$ is 8.5 , the clay systems appear to be gels held together by attractive forces between oppositely charged edge and face of the particles. Evidence for this were provided in the experiment involving TSPP. TSPP when dissolved yields a quadra-valent anion. This ion is very effective at binding to and screening out the positive edge charge of clay particles. Screening this charge completely removessystem yield stress, which would be the case if this edge charge were critical to setting up system microstructure. This is consistent with a "house of cards" description of system microstructure, as is the result of a maximum $\tau_{y}$ at low ion concentration seen in Figs. 1 and 2. The high zeta potential associated with low ion concentration would result in maximum electrostatic attractions between particle edges and faces. At low $\zeta$ the van der Waals attractions dominate the electrostatics, and therefore the yield stress approaches a constant value as $\zeta$ is decreased.

\section{The Link between Micromechanical Bond Rigidity and Macroscopic Shear Moduli}

\section{A. Introduction}

A direct measurement of the particle-particle bond rigidity, $\varkappa_{0}$, between aggregated colloids using a micromechanical three point bending test of assembled linear aggregates employing laser tweezers has been reported, ${ }^{7}$ but it remains to be determined how one may use measured $\varkappa_{\mathrm{o}}$ values to estimate bulk colloidal gel shear moduli, $G$. In

\footnotetext{
${ }^{7}$ J.P. Pantina and E.M. Furst, Langmuir, 22, 5282-5288(2006).
} 
cases where the theory is appropriate, direct measurement of $\varkappa_{\mathrm{o}}$ would appear to allow this estimation to be made regardless of the source of interparticle attractions. This work investigates the comparison of $G$-values computed using the reported $\varkappa_{\mathrm{o}}$ measurements to values obtained by bulk rheometry.

The scaling behavior of the gel modulus, viz., $G \sim \phi^{\mu}$, where $\phi$ is the volume fraction of the particles, has been observed repeatedly over the course of the last few decades. The scaling exponent as well as a prefactor to the scaling law have been derived through a spring model. The spring constant, $\varkappa$, of fractal objects is size dependent. The length scale, $s$, dependence takes the form

$$
\kappa(s)=\kappa_{0}\left(\frac{a}{s}\right)^{\beta},
$$

where $a$ is the particle radius and $\varkappa_{0}$, referred to as the "bond rigidity," is the spring constant between a pair of interacting particles within the cluster. $\varkappa_{0}$ is due to adhesion between particles, and one would expect a large value in relatively strong gels where electrostatic repulsions have been effectively screened, while lower $\varkappa_{0}$-values are expected in weaker gels. The elasticity exponent, $\beta=2+d_{\mathrm{b}}$, where the bond dimension, $d_{\mathrm{b}}$, describes the fractal structure of the gel backbone and must be greater than unity for connectivity. For colloidal suspensions, the critical length scale for gelation, $\xi_{\mathrm{c}}$, representing the average flock size, is exponentially dependent on the flock fractal dimension, $d_{\mathrm{f}}$ in accord with,

$$
\xi_{\mathrm{c}}=a \phi^{1 / 3-d_{\mathrm{f}}} .
$$

The colloidal gel shear modulus is directly related to the flock spring constant as follows,

$$
G=\frac{\kappa\left(\xi_{\mathrm{c}}\right)}{\xi_{\mathrm{c}}}
$$

By substitution, the equation for the modulus then becomes

$$
G=\frac{\kappa_{0}}{a} \phi^{\left(3+d_{\mathrm{b}} / 3-d_{\mathrm{f}}\right)}
$$

The form of the exponential dependence of the modulus on fractal dimension has been 
corroborated through experiment. $^{8}$

Since bond rigidity is now a measurable quantity, Eq. (13) can be fully realized in terms of measurable quantities. Particle radius is routinely measured using many techniques, including light scattering and microscopy. Aggregate fractal dimension is commonly measured with static light scattering, SLS. The bond dimension, $d_{\mathrm{b}}$, has been shown through simulation to be 1.1 for gels made up of diffusion-limited clusters. It is thus possible to calculate $G$ using Eq. (13). Predicting rheological parameters of colloidal gels in terms of the number and strength of particle contacts has been done previously with some success, but these methods depended on the ability to estimate the strength of interparticle attractions, typically according to DLVO theory. As long as aggregates form rigid interparticle bonds, the direct measurement of $\varkappa_{\mathrm{o}}$ now allows for the calculation of bulk properties regardless of the applicability of DLVO theory. Pantina and Furst report $\varkappa_{0}$ data for a series of aqueous linear aggregates with various counterion types and concentrations. They formed linear aggregates of $1.47 \mu \mathrm{m}$ diameter poly(methyl methacrylate) (PMMA) spheres by optical trapping. The aggregates were created in aqueous solutions of $\mathrm{CaCl}_{2}, \mathrm{MgCl}_{2}$, and $\mathrm{NaCl}$ at various concentrations. This allowed them to observe different bond strengths due to varied physicochemical conditions, including the non-DLVO behavior resulting from divalent ion effects.

Micromechanical testing of assembled aggregates is a powerfultechnique allowing for direct access to the strength of interparticle bonding. The present work focuses on one aspect of the application of data measured in this way, viz., predicting bulk mechanical properties of colloidal gels. The objective of this work was thus to explore applicability of micromechanically measured values of $\varkappa_{0}$ to estimating macroscopic gel properties by calculating $G$ and comparing this value with $G$ as measured bybulk rheology.

\section{B. Methods}

Materials were chosen to match the systems used in Pantina and Furst as closely as possible. Gels were prepared by aggregating $1.50 \mu \mathrm{m}$ average diameter spherical

\footnotetext{
${ }^{8}$ R. Buscall, P.D.A. Mills, J.W. Goodwin, D.W. Lawson, J. Chem. Soc., Faraday Trans. 1 84, 4249 (1988).
} 
PMMA latex particles (Bangs Laboratories, Inc., Fishers, IN) which have a reported specific gravity of 1.19. These particles were washed by dilution in deionized water and subsequent centrifuging of the dispersion at $3500 \mathrm{rpm}$ for $15 \mathrm{~min}$ in an International Micro Centrifuge (International Equipment Co., Needham Hts., MA). The wash was repeated until the supernatant was found to have a surface tension equal to that of the deionized water. Three washes proved sufficient. Since these particles are too large to measure aggregate fractal dimension by SLS, it was assumed that $d_{\mathrm{f}}$ developed by aggregation of smaller particles of the same type under identical conditions as the gels studied would be representative of gel $d_{\mathrm{f}}$. Therefore, $60 \mathrm{~nm}$ average diameter PMMA spheres (Bangs Laboratories, Inc.) were used for $d_{\mathrm{f}}$ determination. The salts used to induce aggregation and gelation, $\mathrm{CaCl}_{2}, \mathrm{MgCl}_{2}$, and $\mathrm{NaCl}$, were all Baker Analyzed A.C.S. Reagent grade (J.T. Baker, Inc., Phillipsburg, NJ).

Gel preparation was complicated by the formation of 'armored bubbles', i.e. air bubbles stabilized by the preferential adsorption of particles to the air-liquid interface. Particle stabilized bubbles readily develop upon mixing of these aqueous PMMA suspensions. These bubbles lead to irreproducible gelrheology, and it was therefore necessary to avoid their presence in the gel samples. This need was met by the following sample preparation procedure. The washed particle centrifuge cake was redispersed by physical mixing accompanied by sonication in an Ultrasonic Cleaner (Cole Parmer, Vernon Hills, IL) bath sonicator for $5 \mathrm{~min}$ in enough deionized water so that $\phi<0.1$. Particle stabilized bubbles did occur in low $\phi$ dispersions; however, foaming was greatly reduced. Concentrated stock salt solution was then added to these dilute dispersions, and gelation and sedimentation were allowed to occur over night. The supernatant of these sedimented gels was poured off and the sediment was centrifuged at $3500 \mathrm{rpm}$ for 30 $\min$. The supernatant was again poured off leaving a cake with $\phi \approx 0.45$. This cake was used as the gel sample for shear modulus determination. Exact volume fractions were determined by measuring weight fraction using a simple thermal gravimetric technique, and then calculating $\phi$ from the known particle and solvent densities.

Salt solutions of various concentrations were prepared and filtered through 0.22 m syringe filters (Whatman, Trenton, NJ) into SLS sample cells. The glass cells had been 
soaked in concentrated sulfuric acid and rinsed with filtered water. Drops of concentrated $60 \mathrm{~nm}$ diameter PMMA spheres were added to the salt solutions and subsequently mixed by gently shaking the container by hand. These systems were then allowed to sit overnight. Scattering intensity, $I$, at various scattering angles, $\theta$, was measured for each sample using a BI-200SM goniometer and BI-DS photomultiplier (Brookhaven Instrument Corp., Holtsville, NY) together with a $633 \mathrm{~nm}, 15 \mathrm{mWHeNe}$ laser (Melles Girot, Irvine, CA). Prior to measurement, the index matching fluid, decalin (J.T. Baker, Inc.), was filtered by pumping it through a $0.2 \mu \mathrm{m}$ filter in order to remove dust.

Software automatically collected $I$ versus $\theta$ data and generated plots of $\log I$ versus $\log q$. Where $q$, the scattering vector, is a function of system properties and $\theta$. The negative of the slope of $\log I$ versus $\log q$ plots were then taken to be $d_{\mathrm{f}}$. Each measurement was repeated five times. The fractal dimension measured in this way is an estimate of the true fractal dimension of the rheology samples. If some deviation does exist between the estimate and true fractal dimensions, it is likely that the actual gels, as prepared by the method described above, would tend to be more consolidated than the measured aggregates. This would result in a reduced value for the calculated $G$, where an increase of 0.1 in $d_{\mathrm{f}}$ would result in about a $35 \%$ reduction in $G$.

Gel shear moduli were measured at a Peltier-system controlled temperature of 25.0 $\pm 0.1^{\circ} \mathrm{C}$ with a Modular Compact Rheometer (MCR) 300 (Anton Paar, Ashland, VA). Gel samples were held between 50-mm diameter sand blasted plate and a parallel smooth plate. The surface roughness of the sand blasted plate ensures that slip due to depletion at the tool-sample interface does not affect the measurement. At high frequencies, $\omega$, the storage modulus, $G$, of gels tends to asymptote to some value, and $G_{\lim \omega \rightarrow \infty}^{\prime}=G$. Therefore, in order to most closely approximate the shear modulus, the highest available frequency $(100 \mathrm{~Hz})$ allowed by the instrument was used to measure storage modulus. This is a good approximation as long as the characteristic relaxation time of the gel is much longer than the period of the applied strain; which, at $100 \mathrm{~Hz}$, is generally the case for weak gels [20]. Each run consisted of an increase in oscillation amplitude from 0.01 to $100 \%$ at a constant frequency of $100 \mathrm{~Hz}$. The resulting torque on the sand blasted plate was recorded, and from this value the storage modulus was 
calculated by the instrument automatically. The storage modulus at the lowest measurable amplitude was taken to be the best approximation of the shear modulus. Low amplitude values were used in order to ensure that the linear viscoelastic region was probed.

\section{Results}

Gels prepared as described above resulted in $\phi$ ranging from $0.37-0.47$. Measured values of $d_{\mathrm{f}}$ used in calculating $G$ were between 1.8 and $2.1 \pm 0.1$, where the uncertainty represents one standard deviation calculated from the five individual measurements. Table 2 lists $d_{\mathrm{f}}$ values used in calculating $G$.

Table 2: Summary of aqueous salt media used, together with values of the bond rigidity, $\varkappa_{0}$, and fractal dimension, $d \mathrm{f}$, used for calculation of the gel modulus, $G$

\begin{tabular}{lccc}
\hline & Salt concentration $(\mathrm{mM})$ & $\varkappa(\mathrm{N} / \mathrm{m})$ & $d_{\mathrm{f}}$ \\
\hline $\mathrm{CaCl}_{2}$ & 10 & 0.02 & 2.07 \\
& 50 & 0.04 & 2.08 \\
& 100 & 0.28 & 1.80 \\
& 200 & 0.70 & 2.08 \\
$\mathrm{MgCl}_{2}$ & 400 & 0.21 & 2.00 \\
& 50 & 0.06 & 2.03 \\
& 250 & 0.20 & 2.00 \\
& 375 & 0.31 & 1.95 \\
$\mathrm{NaCl}$ & 500 & 0.61 & 2.05 \\
& 100 & 0.02 & 2.00 \\
& 500 & 0.04 & 1.91 \\
\hline
\end{tabular}

Figs. 4-6 show gel modulus as calculated according to Eq. (13) using Pantina and Furst values of $\varkappa_{0}$, listed in Table 2 , compared with gel modulus measured by conventional

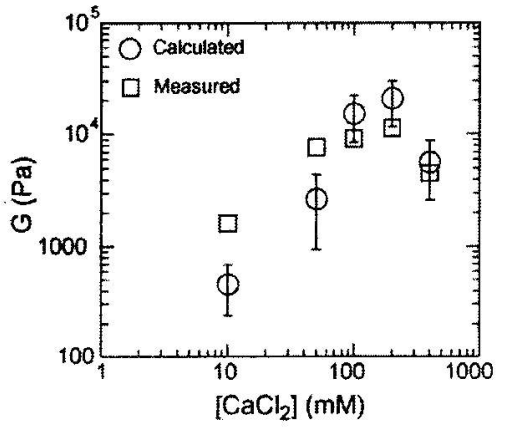

Fig. 4: Log-log plot of calculated and measured $G$ vs. $\mathrm{CaCl}_{2}$ concentration.

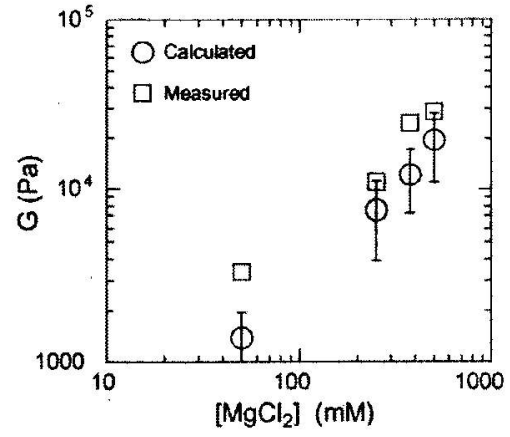

Fig. 5: Log-log plot of calculated and measured $G$ vs. $\mathrm{MgCl}_{2}$ concentration. 
oscillatory rheometry. Error bars on the calculated points are due to the reported uncertainties in $\varkappa_{\mathrm{o}}$ as well as measured uncertainties in $d \mathrm{f}$. In some cases the error bars are large; therefore it does not make sense to attempt to verify the exact values of $\varkappa_{0}$.

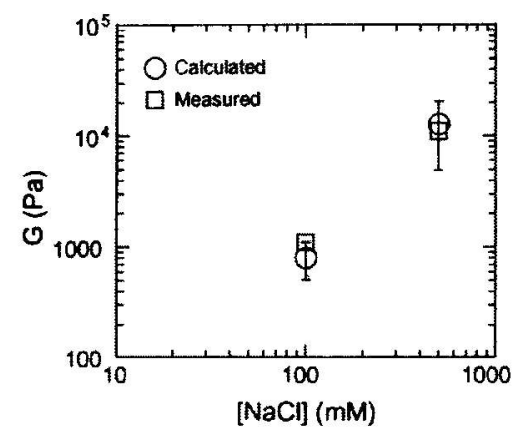

Fig. 6: Log-log plot of calculated and measured $G$ vs. $\mathrm{NaCl}$ concentration.

Despite this limitation it is valuable to compare the data within the given level of certainty. Although some deviation between the calculated and measured values exists, Eq. (13), with the reported values of $\varkappa 0$ can predict $G$ to within a factor of 2 . It is also important to note that both calculated and measured $G$ follow similar trends with salt concentration. Pantina and Furst discuss in particular the effects of divalent ion adsorption, specifically noting that charge reversal occurs above $200 \mathrm{mM} \mathrm{CaCl}_{2}$. This phenomenon causes the observed maximum of Fig. 4. Data for $\varkappa_{\mathrm{o}}$ above the concentration required for charge reversal in $\mathrm{MgCl}_{2}$ systems was not available; therefore, a monotonic trend was expected and is observed in Fig. 5. Divalent ion absorption effects make the usefulness of the direct measurement of $\varkappa_{0}$ clear.

In summary, bulk rheological measurements of colloid gels created from particles and media nearly identical to those used by Pantina and Furst to investigate $\varkappa$ o have been used to explore the link between the microscopic $\varkappa_{\mathrm{o}}$ measurement and macroscopic properties. The data presented attest to the applicability of Pantina and Furst micromechanical determinations of $\varkappa_{\mathrm{o}}$ to the calculation of bulk modulus, $G$. Direct measurement of $\varkappa_{\mathrm{o}}$ thus allows bulk colloidal gel rheology to be predicted within a reasonable degree of certainty, even in cases where DLVO theory breaks down. 


\section{Colloid Aggregation Arrested by Caging within a Polymer Network}

\section{A. Introduction}

The aggregation of colloidal particles within the confines of a polymer network has been studied. An isorefractive covalently cross-linked polymer gel in dimethyl sulfoxide was formulated so that the multicomponent system that is the gel is essentially invisible to light scattering. The high dielectric solvent was chosen so that electrostatics could be used to control the state of aggregation of a colloid dispersed within the gel matrix.

Studies of particles in gels fall into two categories: particle motion in gel media, and systems where particles are used as probes of gel properties. A wealth of knowledge has been gained through experiments with these types

of systems. Many studies of particle dynamics within gels have been reported, including observations of anomalous diffusion, when particles are small compared with the network mesh spacing as well as larger trapped particles fluctuating in space within the network, such as the study described in Part I of this report. The sedimentation behavior of heavy colloids in gel media has been investigated so that it is now well understood that a gel's yielding behavior and elasticity dictate the sedimentation (or creaming) stability of particles dispersed within the gel. Investigations of particle dynamics during gelation have provided an interesting way to probe gelation kinetics. The field of microrheology, beginning with some of the earliest experiments by Freundlich and Seifriz in 1923, who used magnetic particles to probe an aqueous gelatin matrix, deals largely with particles in gels. ${ }^{9}$ Despite these earlier studies, the aggregation behavior of particles within polymer networks remains poorly characterized.

Aggregation in ordinary liquid media, including, mechanisms, kinetics, and resulting aggregate structures has been observed and modeled in great detail. When no potential energy barrier between particles exists, the process is kinetically limited only by particle and cluster diffusion, leading to diffusion limited cluster-cluster aggregation,

\footnotetext{
${ }^{9}$ H. Freundlich and W. Seifriz, Zeitsch. Phys. Chem., 104, 233 (1923);

F. C. MacKintosh and C. F. Schmidt, Curr. Opin. Colloid Interface Sci. 4, 300 (1999).
} 
DLCA, described well by Smoluchowski's population balance equations assuming a constant aggregation kernel.12 Aggregates formed in this way have relatively loose structures, described by a fractal dimension, $d_{\mathrm{f}} \approx 1.8$. In cases where a small barrier exists, close approach of particles or clusters may or may not result in sticking. Such behavior is accurately modeled as 'reaction limited' cluster-cluster aggregation, RLCA, and results in more compact aggregates: $d_{\mathrm{f}} \approx 2.1$. In general, the $d_{\mathrm{f}}$ of colloidal aggregates falls somewhere in the range of 1.8-2.1, depending on the rate-determining step in the aggregation process. ${ }^{10}$ Again, it is unclear how colloid aggregation behavior will be affected by a gel medium.

A key property of polymer networks that is related to aggregation within the network is the characteristic length scale of the density correlation function, $\xi$, often thought of as the mesh spacing of the polymer gel. In a primitive sense, if $\xi$ is smaller than the primary particle dimensions, then particle diffusion and aggregation would not occur. Measurement of the mesh spacing can be complicated but is typically effected using scattering methods. However, a simple estimate of the mesh spacing can also be taken from rubber elasticity theory. ${ }^{11}$ If a polymer gel is thought of as a collection of interconnected entropic springs giving rise to a bulk shear modulus, $G$, then the cross-link density, $\rho_{x}$, is related to the modulus as,

$$
G=A \rho_{x} k_{\mathrm{B}} T,
$$

where $A$ is a constant (equal to unity in the simplest form of the theory), $k_{\mathrm{B}}$ is Boltzmann's constant, and $T$ is the temperature. Then as a rough estimate of the average network spacing, the elastically effective mesh spacing can be defined as,

$$
x_{e}=\sqrt[3]{1 / \rho_{x}}=\sqrt[3]{k_{\mathrm{B}} T / G},
$$

which can be calculated from measurement of the gel shear modulus.

It is hypothesized that clusters of colloids aggregating within a polymer network will grow until they become caged by the network. This caging will effectively arrest the aggregation process in some state depending on the network mesh size. Practically, this

\footnotetext{
${ }^{10}$ A. Y. Kim and J. C. Berg, Langmuir, 16, 2101 (1999).

${ }^{11}$ S. F. Edwards and T. A. Vilgis, Rep. Prog. Phys., 51, 243 (1988).
} 
phenomenon could be taken advantage of in formulating materials where undesired aggregation would normally occur without the gel present. It may be possible to create systems made up of loose gels that can be made to flow easily, yet still hinder aggregation to an acceptable level. It may also be possible to generate aggregates of controlled size which could then be recovered. These possibilities motivate the present work to investigate the aggregation behavior of colloidal particles within a polymer gel network.

\section{B. Methods}

A simple way to model colloid aggregation within a polymer gel is to adjust Smoluchowski's population balance equations to account for the trapping of large aggregates. This means that particles and aggregates smaller than some cutoff related to the spacing within the gel diffuse and aggregate as they would in a purely viscous medium. Then when the aggregates become larger than the spacing within the gel they become immobile. This model predicts an arrested aggregation process producing a final aggregate population that can be used to calculate a light scatting intensity.

Tracking the aggregation process in this way splits particle and cluster interactions into three categories. First, hydrodynamic units that are mobile will diffuse, interact, and combine to form larger units. In the second case, one mobile unit diffuses and interacts with an immobile partner to combine and form one unit. In the third case both units are immobile, so that aggregation is impossible. Smoluchowski's constant aggregation kernel, $k$, may thus be adjusted as follows:

$$
k_{i j}=\left\{\begin{array}{c}
i<x, j<x: k \\
i<x, j \geq x: \frac{1}{2} k \\
i \geq x, j \geq x: 0
\end{array}\right.
$$

where $k_{i j}$ is the rate constant for the process of an aggregate of $i$ particles merging with an aggregate of $j$ particles, and $x$ is the cutoff size, in terms of the number of primary particles, between mobile and immobile aggregates. For calculation purposes, an effective diffusivity, $D$, that reflects a sharp, cluster-size dependent, transition from some finite value for small clusters, $D_{i}(i<x)=D$, to zero for trapped clusters, $D_{i}(i \geq x)=0$, is defined. Then for clusters of radius $a, k$ can be calculated as $k=8 \pi D a$. The conditional 
rate constant can then be used to solve the population balance equations. The rate of change in the population density, $n_{p}$, of aggregates of $p$ particles according to Smoluchowski's population balance is

$$
\frac{d n_{p}}{d t}=\frac{1}{2} \sum_{i=1, i+j=p}^{i=p-1} k_{i j} n_{i} n_{j}-\sum_{i=1}^{i=\infty} k_{i p} n_{i} n_{p} .
$$

A solution to this population balance for $x=3$ is shown in Fig. 7 as a plot of the population densities of aggregates containing 10 particles or less as a function of time. Setting $x=3$ means that any cluster larger than a dimer is immobilized. The initial conditions for this hypothetical system are that the dispersion is made up entirely of individual primary particles, with an initial number density of $n_{1}=10^{19} \mathrm{~cm}^{-3}$.

The solution was arrived at by calculating $\Delta n_{p} / \Delta t$ at time steps that satisfy $k n_{1} \Delta t=$ 0.1 , and assuming a linear function over this small time interval. The solution was not significantly changed by using a smaller interval. Calculations were made over sufficient time steps to ensure that the population densities of the mobile units for a given system reached $1 \mathrm{~cm}^{-3}$. The maximum value for $p$ was found for any $x$ by finding the largest $p$ where $n_{p} \geq 1 \mathrm{~cm}^{-3}$ when the final time step was reached; for $x=3, p_{\max }=35$. It is clear from Fig. 7 that applying these rules to the population balance results in a final state that is a dispersion of small aggregates, in contrast with the case where unhindered aggregation ultimately results in one aggregate of all the particles.

Although real systems are significantly more complicated than described by this model, it is useful to compare its predictions with data. One simplification is that all mobile hydrodynamic units have essentially a constant diffusivity. This is a poor approximation, since in gels the cluster diffusivity is dependent on the relative size scales of the mesh and the cluster. This makes predictions of aggregation kinetics from this model not strictly appropriate; however the predicted final state of aggregation for a given system remains of interest. Probably the most challenging factor to account for is the likely broad range in gel mesh spacing. To model this would mean that $x$ is not some constant but would fall within a range where the relevant value depends on the specific location of the aggregate being accounted for in the population balance. However, if $x$ is allowed to represent an average of the mesh spacing, it is possible to use the model to 
predict the final population distribution as well as the light scattering intensity.

In general, for a dispersion of monodisperse scattering centers the total intensity at any scattering vector $q$ is given by the product of the number of scatterers $n_{0}$ and the intensity from each scattering center $I_{1}$

$$
I(q, t=0)=I_{i}=n_{0} I_{1}
$$

For Rayleigh-Gans-Debye (RGD) scattering, $4 \pi a \theta / \lambda<1 \mathrm{rad}$, where $\theta$ is the scattering angle, and $\lambda$ is the wavelength of light, the intensity from an aggregate of $p$ particles with cluster radius $a$ is given by

$$
I_{p}=p^{2} I_{1}
$$

It is important to note that due to limited cluster growth the hypothetical case of Fig.7 adheres to the RGD criterion throughout the aggregation process as long as the primary particles are sufficiently small, and $\theta$ is sufficiently low, as with the experiments to be described later, where the primary particle radius is $a=10 \mathrm{~nm}$ and $\theta=30^{\circ}$.

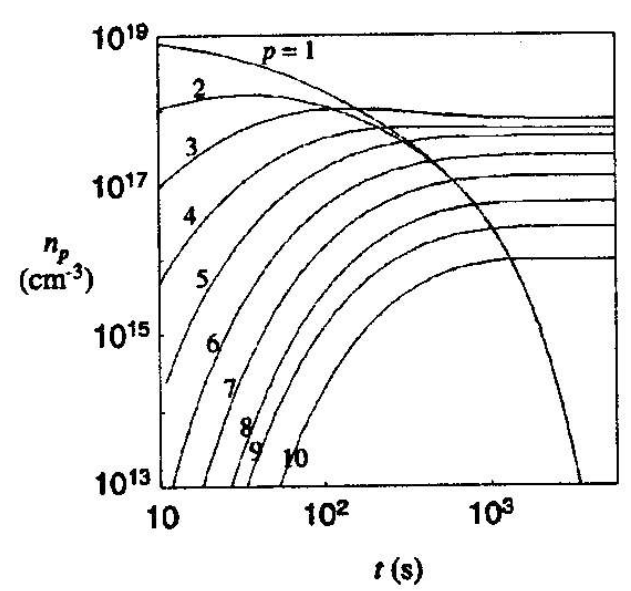

Fig. 7: Computed evolution of the aggregate population in a gel where clusters larger than two primary particles $(p=2)$ are immobile. Aggregates of more than 10 particles $(p>10)$ are predicted and accounted for but are not shown in the plot.

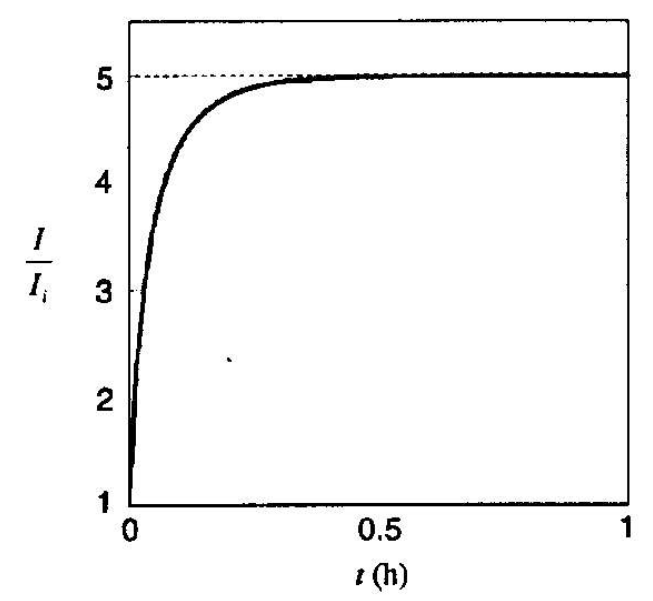

Fig. 8: Predicted scatting intensity increase during the aggregationprocess illustrated in Fig. 7.

The total intensity from a dispersion of clusters at any time $t$ during aggregation is given by $I(t)=\sum_{p} n_{p} I_{p}$. It may be normalized in terms of the measurable initial intensity to 
give

$$
\frac{I(t)}{I_{i}}=\frac{1}{n_{0}} \sum_{p} n_{p} p^{2} .
$$

Combining Eq. (20) with the predictions of the population balance equations gives a prediction of the relative scattering intensity increase during aggregation all the way through arrest, as shownin Fig. 10 for the aggregate population of Fig. 9. The diffusivity of primary particles in a representative gel as measured by photon correlation spectroscopy, $D \approx 10^{-14} \mathrm{~m}^{2} / \mathrm{s}$, was used in the calculations as an estimate of the diffusivity for all mobile clusters. The model predicts that the intensity increases to an asymptotic maximum value which depends on $x$, for $x$ ) $3, I_{\max } / I_{i}=5$.

Gel materials were chosen to satisfy several requirements. First, the solvent had to support electrostatic sol stabilization, so that the state of aggregation of embedded particles could be controlled. Second, the polymer and solvent were required to have indices of refraction, $n$, that were close enough that light scattering intensities from a gel of these two components would be negligible compared to the scattering from embedded particles. Electrostatically stabilized colloids were used in this study because this mode of stabilization is well understood. Such dispersions can be destabilized simply by the addition of sufficient electrolyte, and the resultant aggregation kinetics and aggregate structure, have been well described. Therefore, only solvents that support charge separation were considered for this work which precluded the use of the most common isorefractive gel, viz., cross-linked poly (methyl methacrylate) in toluene. It is difficult to identify an aqueous system that fulfills the isorefractive requirement due to the exceptionally low refractive index of water, $n \approx 1.33$. Water-soluble polymers tend to have indices of refraction that fall in the range $1.4 \leq 1.6$. This means that hydrogels scatter significant amounts of light, making the deconvolution of scattering data from particles intercalated into gels challenging. It was for this reason, as well as the ability to support electrostatic stabilization of dispersions without additives, that a mixture of the high $n$, polar solvents dimethyl sulfoxide(DMSO) and dimethyl formamide (DMF) was used as the solvent in this work. The DMSO used was Fluka Purum grade (g99\% by GC, $\mathrm{H}_{2} \mathrm{O} \leq 0.3 \%$ ) with a measured $\left.n\right) 1.477$ at room temperature $\left(22.5^{\circ} \mathrm{C}\right)$. The DMF (J. T. 
Baker, Phillipsburg, NJ) was Baker analyzed ACS Reagent grade, and its measured refractive index was $n$ ) 1.430 at room temperature. An Abbe refractometer (Erma Optical, Tokyo, Japan) was used to measure the indices of refraction of these solvents. It was found in this work that systems containing polyacrylonitrile (PAN) cross-linked with ethylene glycol dimethacrylate (EGDMA) in DMSO, with a small amount of DMF, are isorefractive. Gels of this combination of materials scatter light with an intensity about twice that of DMSO alone. PAN gels were prepared by free radical polymerization of acrylonitrile (AN) with EGDMA initiated by 2,2'- azobisisobutryronitrile (AIBN).

Gels were prepared within light scattering and rheometry cells so that these types of measurements could be made without disturbing the gel structure. The first step in the gel preparation was to make a stock solution of the initiator. AIBN was dissolved in DMF so that the stock solution concentration was $0.1 \mathrm{~g} / \mathrm{mL}$. EGDMA and AN were then weighed into a glass container. Next, DMSO was added to the cross-linker and monomer mixture. This solution was then split intoa5mLaliquot, which was dispensed into the light scattering cell, and a $35 \mathrm{~mL}$ portion, which was poured into the rheometry cell. In order to remove ambient polymerization quenching $\mathrm{O}_{2}, \mathrm{~N}_{2}$ gas was bubbled through the LS sample for at least $60 \mathrm{~s}$, then the vial was sealed with a septum. The rheometric sample was also sparged with $\mathrm{N}_{2}$ for at least $60 \mathrm{~s}$; the sample container was then sealed with a piece of solid poly (dimethyl siloxane) (PDMS). A small amount of the initiator solution was then added by syringe to each solution. Then both containers were placed into a glass vessel that was purged with $\mathrm{N}_{2}$ and sealed by a ground glass junction and vacuum grease. The vessel was then placed into a $40^{\circ} \mathrm{C}$ oven for $20 \mathrm{~h}$. After gelation, all samples were exposed to ambient atmosphere and allowed to cool to room temperature, thus quenching the reaction. Gels were prepared in this way with a range of formulations. Both the total mole fraction of monomer, $C_{\mathrm{M}}$, and the cross-linker content as a fraction of the total monomer, $C_{\mathrm{X}} / \mathrm{M}$, were varied in order to generate gels of various modulus.

Cerium dioxide, or ceria, particles were provided by Nyacol Nano Technologies (Ashland, MA) with a nominal diameter of $20 \mathrm{~nm}$ and $n \approx 2.2$ were used. Small amounts of the aqueous ceria suspension diluted into DMSO resulted in a positive $\zeta$-potential as measured with a Zeta PALS electrophoretic light scattering system (Brookhaven 
Instruments Corp., Holtsville, NY). These suspensions were stable in terms of aggregation for at least a few months. Although not very soluble, a concentration of 10 $\mathrm{mM} \mathrm{Na} 2 \mathrm{CO} 3$ based on total salt added was found to generate sufficient dissolved ions to induce rapid aggregation of ceria in DMSO. In jar tests, the aggregation was qualitatively similar to the more familiar case of aqueous suspensions. The sol instantly became turbid upon addition of salt solution and the system phase separated by sedimentation over time. A static light scattering (SLS) experiment performed at a series of angles showed that the fractal dimension of ceria aggregated in DMSO by $\mathrm{Na} 2 \mathrm{CO} 3$ was $\approx 1.78$.

Stock ceria suspensions were made by diluting the raw suspension 100x in DMSO. Small amounts of the stock solution were added to the gel reaction solutions before $\mathrm{N} 2$ sparging in order to reach a final volume fraction of $\phi=1.4 \times 10^{-5}$, which is equivalent to a particle number density of $n_{p}=1.7 \times 10^{19} \mathrm{~cm}^{-3}$. These suspensions resulted in multiple orders of magnitude scattering intensity increase over solvent alone. It is on this basis that the gel scattering ( $2 \mathrm{x}$ solvent) was considered negligible in this work.

As an estimate of the shear modulus $G$, the storage modulus $G^{\prime}$ was measured by oscillatory rheometry. As described previously, gel samples were prepared within the measuring cell. A $21 \mathrm{~mm}$ diameter, $16 \mathrm{~mm}$ height, 6 blade vane tool and a $23 \mathrm{~mm}$ diameter cup with a Modular Compact Rheometer (MCR) 300 (Anton Paar, Ashland, VA) were used to perform these measurements. Each test was done at a Peltier controlled temperature of $23.0 \pm 0.1^{\circ} \mathrm{C}$. Frequency sweeps were run at an amplitude of $10 \%$ where the oscillatory frequency, $\omega$, was ramped from $0.1-100 \mathrm{~Hz}$. The modulus reported in this work is $G^{\prime}(\omega=10 \mathrm{~Hz})$. Subsequent amplitude sweeps at $10 \mathrm{~Hz}$, showed that $10 \%$ strain amplitude fell well within the linear viscoelastic region for all gels in this study. A yield stress, $\tau_{y}$, was also determined for each sample by running a shear sweepafter the oscillatory tests. The shear sweep was a rotational experiment where the shear rate was gradually increased from $10^{-3}$ to $10^{3} \mathrm{~s}^{-1}$. An estimate of $\tau_{\mathrm{y}}$ was taken as the shear stress at which the viscosity began to decrease sharply. An experiment was performed in order to investigate the effects of salt addition on the mesh spacing of these gels. A gel was prepared according to the previously described procedure, and its modulus was measured. An amount of $\mathrm{Na}_{2} \mathrm{CO}_{3}$ equivalent to the amount added to the light scattering samples, 
described below, was added, and the sample was aged for $24 \mathrm{~h}$. The modulus was then remeasured. Over this rest period, the salt had no effect on the modulus of this gel.

Aggregation was induced by the addition of salt, in solid form, to the fully formed, particle-laden gels within the light scattering cells. The gel prevented settling but allowed some of the salt to dissolve and diffuse into the sample. The increase in light scattering intensity from aggregating sols was measured by the Zeta PALS instrument with an external goniometer attachment. The external SLS apparatus consisted of a BI-200SM goniometer and BI-DS photomultiplier, both from Brookhaven, together with a $633 \mathrm{~nm}, 15 \mathrm{~mW}$ HeNe vertically polarized laser (Melles Girot, Irvine, CA). For each experiment intensities at $30^{\circ}$ scattering angles were averaged over $100 \mathrm{~s}$ and repeated at 10 min intervals during aggregation.

\section{Results}

The storage modulus increased as the total monomer and crosslinker content of gel reaction solution are increased. This modulus was indicative of the relative cross-linking density within these gels in accord with rubber elasticity theory described by Eq. (15). These gels with a range of moduli should also have a range of mesh spacing where the lowest modulus gel corresponds to the loosest or largest mesh.

The scattering intensity of ceria aggregating within PAN gels was observed over the course of at least $20 \mathrm{~h}$ each for a group of samples. It was found that in some cases $I$ increased until coming to an asymptote after some time, in other cases $I$ did not increase. An example of the asymptotic increase in $I$ is shown in Fig. 9. These types of behavior corresponded with differences in the gel modulus. Samples with moduli above $\sim 80 \mathrm{~Pa}$ did not show any increase in $I$. These samples likely have gel mesh spacing small enough to trap the primary particles and thus prevent any aggregation. At $G=80 \mathrm{~Pa}, x_{\mathrm{e}} \approx 20 \mathrm{~nm}$, from Eq. (15), compared with particle diameters of $\sim 20 \mathrm{~nm}$. This elastically effective mesh size comes close to being predictive of particle trapping. Comparing the time-scales of the intensity increase in Figs. 8 and 9 shows a significant discrepancy. It was noted above that the model is not capable of accurately predicting kinetics. However, the long times observed for aggregation to reach completion in the experimental samples is probably due not only to a decrease in effective diffusivity with cluster growth but also 


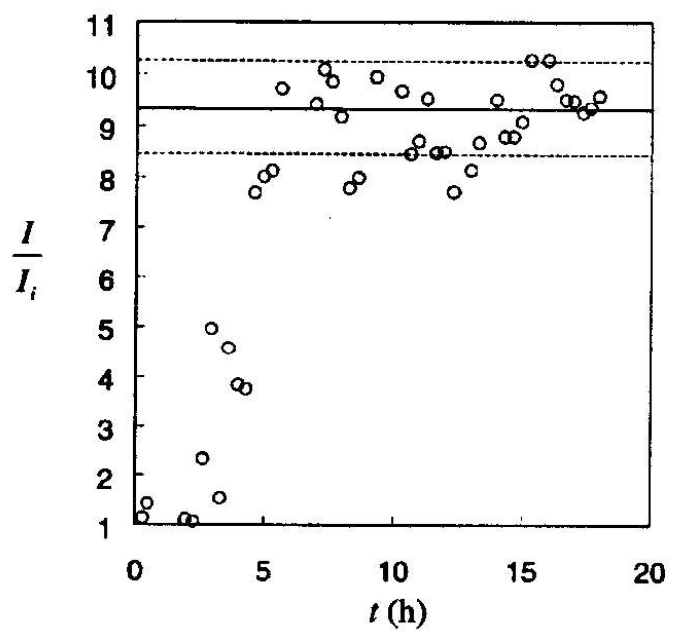

Fig. 9: Scattering intensity increase due to 20 $\mathrm{nm}$ diameter ceria particles aggregating in a 7 mol\% AN, 1.56 mol\% EGDMA/AN gel in DMSO. The solid line represents the normalized scattering intensity averaged between 7 and $20 \mathrm{~h}$, which is taken as the asymptotic maximum increase in intensity due to aggregation, $I_{\max } / I_{i}=9.3$, and the dashed lines represent one standard deviation away from that average.

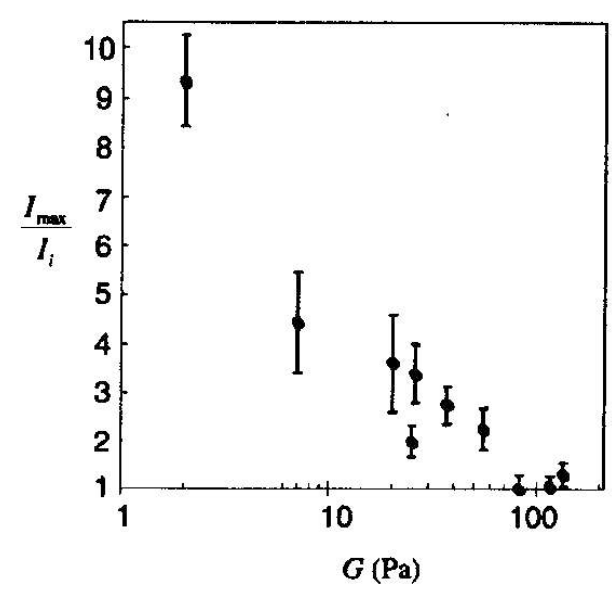

Fig. 10: Final scattering intensity increase for aggregated ceria in gels of various moduli. The error bars represent one standard deviation from the mean maximum scattering intensity.

to the counterion diffusion times. The intensity from all of the samples that did have an increase in $I$ came to an asymptote similar to the case shown in Fig. 9. This indicates that these ceria particles are exhibiting arrested aggregation. Also note that no sedimentation was visually observed, and based on the yield stress of these gels and the force of gravity on the small aggregates, none was expected. This set of samples showed roughly that lower modulus gels resulted in greater $I$ increase, as is shown in Figure 10.

With respect to comparison with the model, the largest $I_{\max } / I_{i}$ observed in this work was $\sim 9.3$, close to the value from the arrested aggregation model for $x=5$ where $I_{\max } / I_{i}=9$. This could be thought of as a model-fitting method for approximating the average mesh spacing within a gel. This sample had a measured $G^{\prime}=2 \mathrm{~Pa}$, which corresponds to $x_{e}=100 \mathrm{~nm}$. In an effort to match $x$ with $x_{e}$ it can be shown that $x_{e}=5 a$ should lead to $x \approx 18$ assuming that the appropriate $d_{f}=1.8$ and that $P=\left(a_{p} / a\right)^{d f}$, where $a_{p}$ is the radius of an aggregate of $p$ particles. Clearly some difference remains. Although it is recognized that $x_{e}$, as calculated from measured modulus values, is a fairly rough approximation, $G^{\prime}$ is used here as an indicator of relative mesh spacing in 
comparing gels. The data in Fig. 10 do suggest that lower-modulus gels allow a greater extent of aggregation than do higher-modulus gels.

In summary, this work hasshown that polymer networks can be used to arrest colloidal aggregation. Aggregation within gels was modeled using an adjusted Smoluchowski population balance model that was used to predict scattering intensities during the process. Scattering intensities from aggregating particles in gels of various moduli were compared with the model. The model predicts, and the data show, that aggregation is arrested by caging in the gel network. Furthermore, it is shown that control over the degree of aggregation may be exerted through gel formulation. It is demonstrated that loose gels allow a greater extent of aggregation than stiffer, higher modulus, gels. This interesting phenomenon could find use in creating aggregates of controlled size or dispersions of systems that would normally aggregate but are limited to some acceptable level of aggregation by the gel matrix.

\section{Summary of Deliverables}

This grant produced one $\mathrm{PhD}$ degree, and the attendant $\mathrm{PhD}$ dissertation. It also resulted in four publications in technical journals and two presentations at national technical meetings. These are documented below.

$\underline{\text { PhD degree awarded }}$

Recipient: Dr. Peter B. Laxton

Date: April 22, 2008

\section{$\underline{\text { PhD Dissertation }}$}

"Linking the Macroscopic Rheological Properties of Gels to Micro= and Nano-scopic Colloidal Phenomena"

by Peter B. Laxton

\section{$\underline{\text { Publications }}$}

1. Laxton, P. B., and Berg, J. C., "Gel Trapping of Dense Colloids," J. Colloid Interface Sci., 285, 152-157 (2005). 
2. Laxton, P. B., and Berg, J. C., "Relating Clay Yield Stress to Colloidal Parameters," J. Colloid Interface Sci., 296, 749-755 (2006).

3. Laxton, P. B., and Berg, J. C., "Investigation of the Link between Micromechanical Interparticle Bond rigidity Measurements and Macroscopic Shear Moduli of Colloidal Gels," Colloids Surfaces A, 301, 137-140 (2006).

4. Laxton, P. B., and Berg, J. C., "Colloid Aggregation Arrested by Caging within a Polymer Network," Langmuir, 24, $9268-9272$ (2008).

\section{Presentations}

1. Paper \#404: 78th ACS Colloid and Surface Science Symposium, Yale University, New Haven, CT June 20-23, 2004: "Gel Trapping of Colloids," by. P. B. Laxton and J. C. Berg.

2. Paper \#385: 80th ACS Colloid and Surface Science Symposium, University of Colorado, Boulder, CO June 18-21, 2006: "Relating Colloidal Aggregate Structure to Rheological Behavior," by. P. B. Laxton and J. C. Berg. 